\title{
Prophylactic Use of Nebulized Hypertonic Saline in Mechanically Ventilated Children: A Randomized Blinded Pilot Study
}

\author{
Steven L Shein MD, John T Gallagher MPH RRT-NPS, \\ Kathleen M Deakins MHA RRT-NPS FAARC, and Dayna M Weinert MD
}

\begin{abstract}
BACKGROUND: Mucolytic agents, such as nebulized hypertonic saline, may improve airway clearance and shorten the duration of mechanical ventilation, but prospective blinded studies in children undergoing mechanical ventilation are lacking. METHODS: Children $<18$ y old who had been intubated for $<12 \mathrm{~h}$ and had an expected duration of mechanical ventilation of $>48$ additional h were prophylactically given $3 \mathrm{~mL}$ of either nebulized hypertonic saline or placebo ( $0.9 \%$ saline) 4 times/d. The primary outcome was duration of mechanical ventilation. Ventilator parameters and the presence of wheezing were recorded before and after study drug administration. RESULTS: The duration of mechanical ventilation was significantly longer in children treated with hypertonic saline (208.1 [interquartile range $136.3-319.8$ ] h) versus those treated with placebo (129.5 [interquartile range $74.4-146.1] \mathrm{h})(P=.03$ by Wilcoxon rank-sum test). After adjusting for baseline levels of PEEP, the duration of mechanical ventilation did not differ between groups. Mechanical ventilation parameters, including dead space and dynamic compliance, did not differ between measurements taken before study drug administration versus measurements taken after. New onset wheezing following study drug administration was rare (1.0\% with hypertonic saline vs $3.0 \%$ with placebo, $P=.36$ by chi-square test). CONCLUSIONS: Administering prophylactic nebulized hypertonic saline to mechanically ventilated children did not improve clinically relevant outcomes, including duration of mechanical ventilation. Wheezing after hypertonic saline treatment was rare. Key words: pediatric; hypertonic saline; mechanical ventilation. [Respir Care 2016;61(5):586-592. (C) 2016 Daedalus Enterprises]
\end{abstract}

\section{Introduction}

Minimizing the duration of mechanical ventilation is a common pediatric ICU goal, since prolonged mechanical ventilation duration is associated with increased morbidity and mortality. ${ }^{1,2}$ Prolonged duration of mechanical venti-

\footnotetext{
Dr Shein is affiliated with the Division of Critical Care Medicine, Rainbow Babies and Children's Hospital, and the Department of Pediatrics, Case Western Reserve University School of Medicine, Cleveland, Ohio. Mr Gallagher and Ms Deakins are affiliated with the Division of Respiratory Care, Rainbow Babies and Children's Hospital, Cleveland, Ohio. Dr Weinert is affiliated with the Division of Radiology, Rainbow Babies and Children's Hospital, and the Department of Radiology, Case Western Reserve University School of Medicine, Cleveland, Ohio.
}

Mr Gallagher has disclosed relationships with Draeger Medical, Mallinckrodt Pharmaceuticals, and Discovery Labs. Ms Deakins has disclosed relationships with Masimo and Hill-Rom. The other authors have disclosed no conflicts of interest. lation may worsen clinical outcomes by several mechanisms, including ventilator-induced lung injury, prolonged usage of sedative and analgesic medications, and ventilator-associated pneumonia. In one cohort, survival to pediatric ICU discharge was $\sim 94 \%$ among children mechanically ventilated for $<96 \mathrm{~h}$ but was $<80 \%$ among children with longer durations of mechanical ventilation. ${ }^{2}$

Mechanically ventilated patients may have ineffective airway clearance due to multiple mechanisms, including

\footnotetext{
Dr Shein presented a version of this paper at the Society of Critical Care Medicine's 44th Critical Care Congress, held January 17-21, 2015, in Phoenix Arizona.

Correspondence: Steven L Shein MD, 11100 Euclid Avenue, RB\&C 3rd Floor, Pediatric Critical Care Medicine, Cleveland, $\mathrm{OH}$ 44106. E-mail: steven.shein@uhhospitals.org.
}

DOI: $10.4187 /$ respcare.04418 
weakened expiratory muscles, ineffective cough, and decreased mucociliary function..$^{3,4}$ This can lead to obstructive airway secretions, which may be especially tenacious due to conservative fluid management. ${ }^{3,5}$ Obstructive airway secretions may prolong mechanical ventilation by causing atelectasis, inadequate gas exchange, and endotracheal

\section{See the Related Editorial on Page 716}

tube obstruction, with resultant cardio-respiratory instability. Secretions may induce coughing, leading to increased sedative usage, and contribute to patient-ventilator asynchrony. Mucolytic therapies, such as dornase and nebulized hypertonic saline, are used in diseases like cystic fibrosis and bronchiolitis to minimize obstructive secretions by decreasing mucus viscosity and facilitating airway clearance. ${ }^{6,7}$ These medications may similarly improve clinical outcomes in mechanically ventilated children, but data are limited. In the only available study of prophylactic mucolytic therapy in mechanically ventilated children, subjects $<2$ y old recovering from cardiac surgery randomized to dornase alfa therapy had a significantly decreased duration of mechanical ventilation versus those given saline placebo ( $52 \mathrm{~h}$ vs $82 \mathrm{~h}$ ). ${ }^{8}$ This suggests that all children undergoing mechanical ventilation may benefit from airway clearance therapy, probably related to their smaller airways, less developed musculature, and increased sedation requirements. Further study is needed to either confirm that such therapies should be provided in a prophylactic manner to all children undergoing mechanical ventilation or establish that only patients with evidence of significant secretion retention should be treated.

Hypertonic saline may be even more effective, since mechanically ventilated newborns with persistent atelectasis had greater improvement in radiographic findings and oxygen saturation when randomized to receive hypertonic saline compared with dornase alfa. ${ }^{9}$ There are multiple possible advantages of hypertonic saline compared with dornase alfa. Dornase alfa, which hydrolyzes long strands of DNA, is effective only in patients who have leukocytes or bacteria present in tracheal aspirates. ${ }^{10} \mathrm{Hy}-$ pertonic saline may be effective even if those conditions are not present, because it works as both a mucolytic (by disrupting ionic bonds in mucus) and an expectorant (by promoting water secretion into the airway by inducing a hyperosmolar state). ${ }^{11-13}$ Hypertonic saline may also decrease biofilm formation, increase levels of antioxidant thiols, and promote the actions of endogenous mucolytics. ${ }^{12,14}$ It is also less expensive than dornase alfa. However, prior prospective studies of prophylactic nebulized hypertonic saline in mechanically ventilated children are lacking. In this paper, we report the findings of a pilot study of prophylactic nebulized hypertonic saline admin-

\section{QUICK LOOK}

\section{Current knowledge}

Mechanically ventilated patients often have ineffective airway clearance, and children are especially prone to obstructive secretions. Mucolytic agents like dornase alfa and hypertonic saline may mitigate airway obstruction. Prophylactic administration of dornase alfa shortened ventilation duration in children $<2$ y old in a prior randomized controlled trial, but prophylaxis with nebulized hypertonic saline has not been evaluated in ventilated children.

\section{What this paper contributes to our knowledge}

After adjusting for disease severity, prophylactic administration of nebulized hypertonic saline was not associated with a significant difference in duration of ventilation in this pilot study. Post-treatment wheezing was found to be a rare event.

istered to children undergoing mechanical ventilation. We hypothesized that the duration of mechanical ventilation would be significantly decreased in children given prophylactic nebulized 3\% hypertonic saline versus children given saline placebo.

\section{Methods}

The institutional review board of the University Hospitals of Cleveland approved this study, which was conducted between October 2013 and May 2014. Informed consent was obtained from a parent/guardian of all participants. Children $<18$ y old with an expected duration of mechanical ventilation of $>48$ additional $\mathrm{h}$ cared for in the pediatric ICU of Rainbow Babies and Children's Hospital were eligible for the study. Exclusion criteria were participation in another clinical trial; cystic fibrosis; status asthmaticus; pulmonary hemorrhage; pulmonary contusion; home use of positive-pressure ventilation or oxygen therapy; preexisting tracheostomy; allergy to nebulized hypertonic saline, saline, or albuterol; and previous prescription of mucolytic therapy by the clinical team during this hospitalization. Children were only eligible for enrollment if $<12 \mathrm{~h}$ had elapsed since endotracheal intubation.

Randomization and study drug blinding was performed by the University Hospitals of Cleveland Pharmacy Department's Investigational Drug Service; all other parties (investigators, clinicians, families, etc) were blinded to group allocation. Subjects were randomized to receive either $3 \mathrm{~mL}$ of $3 \%$ hypertonic saline or $3 \mathrm{~mL}$ of $0.9 \%$ saline as a study drug. The study drug was administered 4 times/d by nebulization via the Solo nebulizer (Aerogen, 
Galway, Ireland). The nebulizer was adapted to fit within a disposable flow-inflating resuscitation bag appropriately sized for each subject. For each administration of the study drug, a respiratory therapist temporarily suspended mechanical ventilation and subsequently manually ventilated the subject with the flow-inflating bag/nebulizer apparatus. Albuterol $(2.5 \mathrm{mg})$ co-administration was mandated only for those children who had wheezing following a prior dose of the study drug. The study drug was continued until extubation or a maximum of $7 \mathrm{~d}$ of treatment. Study drug administration was deferred if the child was concurrently hemodynamically unstable per the clinical team or on high-frequency oscillatory ventilation, $\mathrm{F}_{\mathrm{IO}_{2}}>0.75$ or PEEP $>11 \mathrm{~cm} \mathrm{H}_{2} \mathrm{O}$. The Evita XL (Dräger, Telford, Pennsylvania) was used exclusively for conventional ventilation.

Demographics, baseline sodium level, and indication for mechanical ventilation were collected at enrollment. Pediatric Risk of Mortality III score was calculated. ${ }^{15}$ Ventilator parameters (mandatory rate, peak inspiratory pressure [PIP], tidal volume [mL/kg], PEEP, $\mathrm{F}_{\mathrm{IO}_{2}}$ ), dead space (measured by the ventilator), end-tidal partial pressure of $\mathrm{CO}_{2}, \mathrm{~S}_{\mathrm{pO}_{2}}$, and the presence of wheezing were documented preceding study drug administration and again within $60 \mathrm{~min}$ following administration. When calculating the duration of mechanical ventilation, extubation was considered successful if re-intubation did not occur within the subsequent $24 \mathrm{~h}$. The change in blood sodium level was calculated as the difference between the mean post-enrollment sodium level during ICU care and the sodium level at enrollment. A radiologist (DMW) blinded to treatment allocation scored the first chest radiograph of each of the first $7 \mathrm{~d}$ of the study using a previously described system. Briefly, for each of 5 pulmonary lobes, linear atelectasis $=1$ point, subsegmental atelectasis $=2$ points, and lobar atelectasis $=3$ points (total range: $0-15$ points). ${ }^{10}$

A Data Safety Monitoring Board oversaw the study. A priori determined stopping rules were: (1) wheezing after study drug administration despite albuterol co-administration that was associated with an increase in endtidal partial pressure of $\mathrm{CO}_{2}$ of $>15 \mathrm{~mm} \mathrm{Hg}$ from baseline; (2) a desaturation episode within $30 \mathrm{~min}$ of study drug administration to $\mathrm{S}_{\mathrm{pO}_{2}}<80 \%$ that did not resolve within $2 \mathrm{~min}$; and (3) a serum sodium level that was both $>150 \mathrm{mEq} / \mathrm{L}$ and increased $\geq 10 \mathrm{mEq} / \mathrm{L}$ from baseline.

Differences between treatment groups were analyzed using the Wilcoxon rank-sum test (continuous variables) and Fisher exact or chi-square test (categorical variables). Variables associated with the primary outcome, duration of mechanical ventilation, were determined using Pearson correlation (continuous variables) and Wilcoxon rank-sum test or Kruskal-Wallis analysis of variance (categorical). Variables nearly associated with duration of mechanical ventilation $(P<.10)$ were included in a multivariable linear regression analysis. Bonferroni correction was used

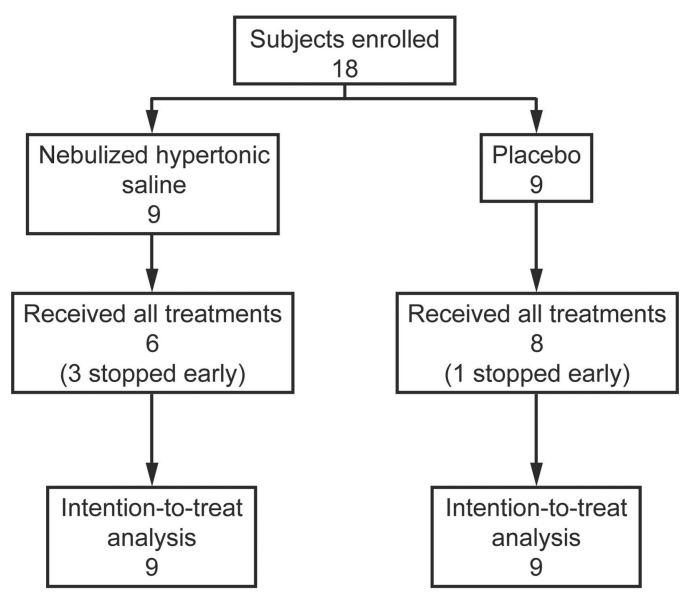

Fig. 1. Flow chart.

to account for multiple variables, and $P<.05$ was considered significant. All analyses were done using SigmaPlot 12.5 (Systat Software, San Jose California). Data are shown as $n(\%)$ or median (interquartile range [IQR]).

\section{Results}

Eighteen children were enrolled in the study (Fig. 1). Median age was 17.9 (5.0-44.0) weeks, and 5 (28\%) were female. Most subjects were either African-American (9 [50\%]) or white (8 [44.4\%]), and most (12 [66.7\%]) were mechanically ventilated due to an acute respiratory infection (eg, pneumonia, bronchiolitis). Indications for mechanical ventilation in other subjects were neurologic injury (spinal muscular atrophy [hypertonic saline] and traumatic brain injury [placebo]), postoperative recovery (otolaryngological [hypertonic saline] and cardiac [placebo]), diaphragmatic hernia, and shock. There were no significant differences in demographics, prevalence of respiratory infection, Pediatric Risk of Mortality III score, or number of doses of the study drug administered between treatment groups (Table 1).

In univariate analysis, the duration of mechanical ventilation was significantly longer in children treated with prophylactic hypertonic saline (208.1 [IQR 136.3-319.8] h) versus those treated with placebo (129.5 [IQR 74.4146.1] h) $(P=.03)$ (Table 2). Weight, age, sex, race, and respiratory infection were not associated with the duration of mechanical ventilation (all $P>.20$ ), but PEEP at the time of the first study drug administration was associated with mechanical ventilation duration $(P=.039)$. After adjusting for baseline PEEP, there was no significant difference in duration of mechanical ventilation between the 2 treatment groups. There were also no significant differences in the duration of ICU care, hospital stay, or the rate of re-intubation between the 2 treatment groups. 
Table 1. Demographics

\begin{tabular}{lccr}
\hline \hline & HTS & Placebo & $P$ \\
\hline Subjects, $n$ & 9 & 9 & \\
Age, median (IQR) wks & $12.7(5.6-32.6)$ & $26.0(3.1-174.2)$ & .66 \\
Weight, median (IQR) kg & $5.3(3.6-7.8)$ & $7.9(3.6-10.2)$ & .66 \\
Female, $n$ (\%) & $2(22 \%)$ & $3(33 \%)$ & $>.99$ \\
Race, $n$ (\%) & & & .20 \\
$\quad$ African-American & 5 & 4 & \\
$\quad$ Caucasian & 3 & 5 & \\
$\quad$ Other & 1 & 0 & .93 \\
Respiratory infection, $n(\%)$ & $6(67 \%)$ & $6(67 \%)$ & .72 \\
PRISM score, median (IQR) & $5(3.5-9.0)$ & $5(3.0-8.5)$ & \\
Drug doses/subject, & $20(5-28)$ & $19(9.5-20)$ & \\
$\quad$ median (IQR) & & & \\
& & & \\
There were no significant differences between treatment groups by chi-square, Fischer exact, \\
or Wilcoxon rank-sum tests. \\
IQR = interquartile range \\
HTS $=3 \%$ hypertonic saline \\
PRISM = Pediatric Risk of Mortality III
\end{tabular}

Table 2. Outcomes

\begin{tabular}{lccc}
\hline \hline & HTS & Placebo & $P$ \\
\hline $\begin{array}{l}\text { Duration of mechanical } \\
\text { ventilation, }\end{array}$ & $208.1(136.3-319.8)$ & $129.5(74.4-146.1)$ & .03 \\
$\quad$ median (IQR) h & & & \\
$\begin{array}{l}\text { Duration of ICU care, } \\
\text { median (IQR) d }\end{array}$ & $12(9-17)$ & $8(6-12.5)$ & .08 \\
$\begin{array}{l}\text { Hospital length of stay, } \\
\text { median (IQR) d }\end{array}$ & $17(16-22.5)$ & $15(9-22.5)$ & .45 \\
$\begin{array}{l}\text { Re-intubation, } n \\
\text { In these unadjusted analyses, hypertonic saline was associated with an increased duration of } \\
\text { mechanical ventilation. Other outcomes did not differ significantly between groups. }\end{array}$ & \\
HTS = 3\% hypertonic saline \\
IQR $=$ interquartile range
\end{tabular}

Baseline chest radiograph scores were 6 (IQR 4.5-9.0) points in the hypertonic saline group and 4 (IQR 2.5-6.5) points in the placebo group, which approached a statistically significant difference $(P=.09)$ (Fig. 2). There was no significant difference between baseline radiographic score and the mean post-enrollment radiograph score (4 [IQR 2.3-5.8] for hypertonic saline; 4.7 [IQR 3.9-6.0] for placebo $)$ in either treatment group $(P=.72$ for hypertonic saline, $P=.18$ for placebo).

There were no significant differences in mechanical ventilation parameters measured after study drug administration versus measurements taken before study drug administration in either group (Fig. 3). Several parameters measured before hypertonic saline therapy differed significantly versus measurements taken before placebo therapy, including lower dynamic compliance and higher rate, PEEP, $\mathrm{F}_{\mathrm{IO}_{2}}$, and dead space. Wheezing (Table 3) was also more

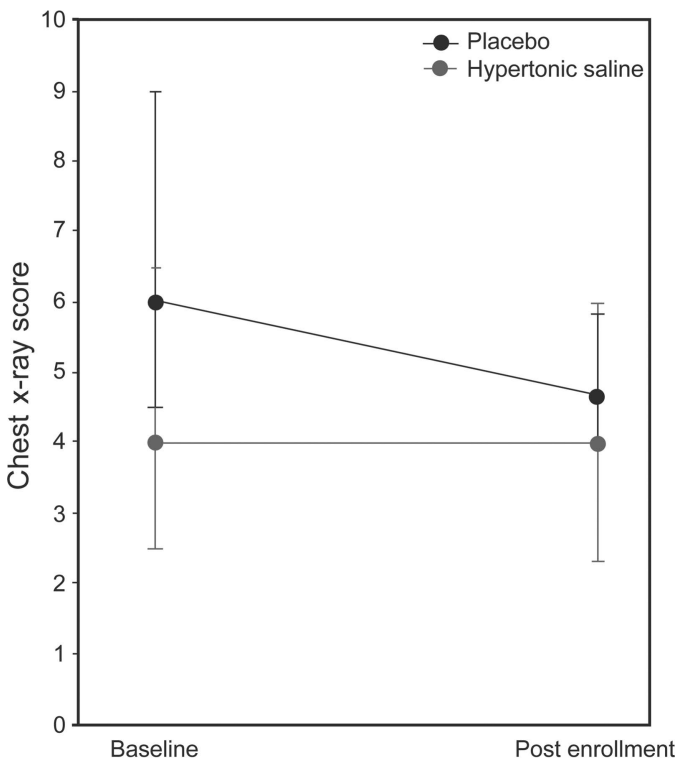

Fig. 2. Chest radiograph scores at baseline and the average of all post-enrollment scores. Scores ranged from 0 to 3 for each of the 5 lobes of the lungs ( 15 is the worst possible cumulative score). Scores did not differ significantly in either group between baseline and post-enrollment. The difference between baseline levels between groups approached significance $(P=.09)$. Points represent median; error bars denote interquartile range.

prevalent before hypertonic saline (8.3\%) than before placebo $(0 \%)(P<.001)$. However, development of wheezing following study drug administration was rare, and its prevalence did not differ between groups $(1.0 \%$ with hypertonic saline vs $3.0 \%$ with placebo, $P=.36$ ). Baseline sodium level and the change in sodium levels also did not differ between groups. Four children exited the study early ( $P=.58$ between groups). Two children met the second a priori stopping rule, and 2 were removed due to coughing (one by an attending physician and one by a parent). No subject met the first or third stopping rule.

\section{Discussion}

Administering prophylactic nebulized 3\% hypertonic saline to mechanically ventilated children was not associated with shorter duration of mechanical ventilation versus placebo in this randomized blinded pilot study. Similarly, there were no salutary effects on mechanical ventilation parameters or chest radiographic scores. Our data do suggest that nebulized hypertonic saline may be administered to mechanically ventilated children with minimal risk of inducing wheezing or hypernatremia but may be associated with transient hypoxemia in some children.

Previously, it has been shown that administering dornase, another mucolytic agent, may reduce the duration of mechanical ventilation in children recovering from cardiac surgery. ${ }^{8}$ Though there are no prior studies of prophylactic 


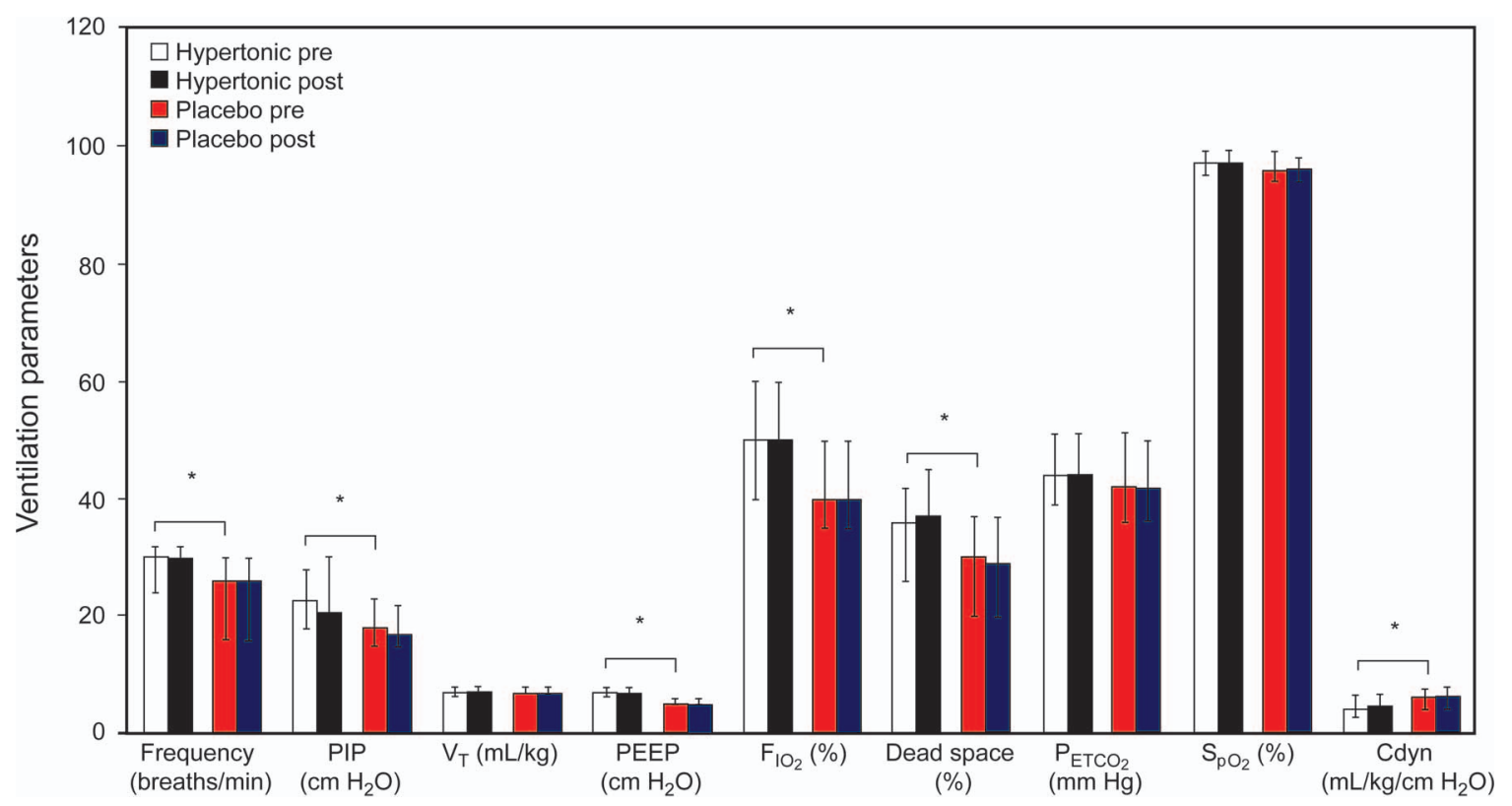

Fig. 3. Mechanical ventilator parameters before and after drug administration. There were no differences between the before and after values in either group. Several parameters differed before drug administration between groups, suggesting worse respiratory mechanics in the hypertonic saline group. Data are shown as median; error bars denote interquartile range. Cdyn = dynamic compliance of the respiratory system; $\mathrm{PIP}=$ peak inspiratory pressure; $\mathrm{V}_{\mathrm{T}}=$ tidal volume; $\mathrm{P}_{\mathrm{ETCO}_{2}}=$ end-tidal $\mathrm{CO}_{2} .{ }^{*}, \mathrm{P}<.05$.

Table 3. Adverse Effects

\begin{tabular}{lccc}
\hline \hline \multicolumn{1}{c}{ Effect } & 3\% Hypertonic Saline & Placebo & $P$ \\
\hline Wheezing prior to drug, $n(\%)$ & $12 / 144(8.3)$ & $0 / 135(0)$ & $<.001$ \\
Developed wheezing following drug, $n(\%)$ & $1 / 105(1.0)$ & $3 / 101(3.0)$ & .36 \\
Baseline $\left[\mathrm{Na}^{+}\right]$, median (IQR) $\mathrm{mEq} / \mathrm{L}$ & $140(132-141.5)$ & $138(135-143)$ & .79 \\
Change in $\left[\mathrm{Na}^{+}\right]$, median $(\mathrm{IQR}) \mathrm{mEq} / \mathrm{L}$ & $-1.5(-4.1$ to 7.1$)$ & $1(-0.9$ to 6.7$)$ & .22 \\
Withdrawn from study, $n(\%)$ & $3(33.3)$ & $11.1)$ & .58
\end{tabular}

Wheezing prior to study drug was more common in the hypertonic saline group, but there were no differences in the development of wheezing after study drug between groups. There were no significant differences in other adverse effects by chi-square, Fisher exact, or Wilcoxon rank-sum tests.

$\mathrm{IQR}=$ interquartile range

nebulized hypertonic saline in pediatric subjects undergoing mechanical ventilation, a prospective randomized study of neonates with atelectasis reported better radiographic outcomes with hypertonic saline versus dornase alfa. ${ }^{9}$ This may reflect the differing mechanisms of hypertonic saline and dornase alfa, since hypertonic saline will decrease mucus viscosity even in the absence of high-DNA content mucus and therefore be beneficial in a wider population. We selected hypertonic saline as our study drug because we thought it was more likely to be efficacious in a heterogeneous population like children undergoing mechanical ventilation in a medical/surgical pediatric ICU.

There are probably several possible reasons why our data do not show improved duration of mechanical ventilation with hypertonic saline prophylaxis. First, our sample size $(\mathrm{N}=18)$ was smaller than the prior prospective neonatal $(\mathrm{N}=40)$ or postoperative cohorts $(\mathrm{N}=100)$, and our study may not have been adequately powered to detect a statistically significant effect on mechanical ventilation duration. ${ }^{8,9}$ However, there is no trend toward benefit observed in our data to suggest that this is the case. Second, our population probably represents a more heterogeneous cohort than either neonates or cardiac surgery patients. Adults undergoing mechanical ventilation are likely to be similarly heterogeneous, and hypertonic saline did not improve oxygenation, radiographs, or duration of mechanical ventilation in a pilot study of that cohort either. ${ }^{16}$ Future studies may find a benefit if a more homogenous population is selected, although the results would be less generalizable. Finally, despite randomization, illness severity appeared different at baseline. Children given hypertonic saline had significantly more unfavorable radiographic findings and pulmonary mechanics at enrollment. Importantly, mechanical ventilation duration did not differ sig- 
nificantly between groups after controlling for baseline PEEP as a marker of illness severity. Similar reasons probably explain why our data do not corroborate prior evidence that hypertonic saline improves radiographic scores. ${ }^{9}$

If hypertonic saline led to acute decreases in airway obstruction, we would have expected to see improvements in oxygenation and/or dynamic compliance of the respiratory system. Our data do not suggest that either of these occurred. This differs from the findings of a prospective study of 3\% hypertonic saline in neonates with atelectasis, where $\mathrm{S}_{\mathrm{pO}_{2}}$ improved by $4.6 \pm 0.8 \%$ following treatment. However, our findings are similar to those of a retrospective study of neonates given $7 \%$ hypertonic saline, in which there were no statistically significant improvements in breathing frequency, $\mathrm{PIP}, \mathrm{F}_{\mathrm{IO}_{2}}$, or $\mathrm{P}_{\mathrm{aCO}_{2} \cdot{ }^{17}}$

Although our data do not support routine use of hypertonic saline in mechanically ventilated children, survey data show that hypertonic saline is prescribed routinely during mechanical ventilation by a large number of pediatric ICU physicians. ${ }^{18}$ Our study provides these practitioners with previously unavailable data pertaining to adverse effects of hypertonic saline in children undergoing mechanical ventilation. We are unaware of any prior prospective data reporting the incidence of post-hypertonic saline wheezing in ventilated children. Because hypertonic saline may induce bronchospasm in patients with asthma, bronchodilators are often co-administered with (or administered before) hypertonic saline, even to subjects without asthma. ${ }^{19}$ One prior retrospective study of spontaneously breathing bronchiolitis subjects reported rare wheezing with hypertonic saline, but those data were based on clinically documented adverse event reports. ${ }^{20}$ In our study, we prospectively auscultated before and after hypertonic saline nebulization and found that wheezing developed after hypertonic saline in only $1.0 \%$ of treatments. This strongly suggests that concurrent bronchodilator treatment is not needed for all children treated with hypertonic saline, although confirmatory data are needed. Similarly, our data suggest that nebulized hypertonic saline does not affect serum sodium levels, as has also been reported in neonates. ${ }^{9}$ Two subjects were removed from the study because of desaturation episodes. Similar infrequent episodes have also been reported when hypertonic saline is given to neonates. ${ }^{17} \mathrm{We}$ suggest that practitioners monitor for this closely if hypertonic saline is used and be prepared to provide manual ventilation with increased $\mathrm{F}_{\mathrm{IO}_{2}}$ and suctioning. Pre-oxygenating before hypertonic saline should also be considered.

Although we are unaware of any other prospective randomized controlled trial of hypertonic saline in mechanically ventilated pediatric ICU subjects, our study does have limitations. As mentioned above, our sample size was small, heterogeneous, and probably underpowered. This project was intended from the onset to be a pilot study and was approved by the institutional review board as such. Pilot studies are routinely not designed to be sufficiently powered, although our sample size $(\sim 20)$ was selected a priori because it was powered to detect a 1.2-d difference in mechanical ventilation duration (as reported by Riethmueller et al) ${ }^{8}$ if the SD value of our mechanical ventilation duration had been $\sim 1 \mathrm{~d}$. Despite our small sample size, no prior data regarding prophylactic nebulized hypertonic saline are available. Our sample size was insufficient to allow for meaningful subgroup analyses. Investigators were not available within $12 \mathrm{~h}$ of intubation for all children who would have otherwise met inclusion criteria, so some potential subjects were missed. Pre- and post-treatment data were collected by the clinical team, and some entries were missed. All subjects were cared for in a single center. It is possible that nebulized normal saline may have salutary effects and that use of a different placebo (eg, air, water, etc) might have allowed us to identify benefits of hypertonic saline therapy. We did not measure mucus viscosity or content (eg, cell count) and were unable to evaluate for associations between mucus characteristics and hypertonic saline efficacy. It is also possible that alternative inclusion criteria, such as evidence of retained secretions or more severe lung disease, could have identified a population where hypertonic saline therapy is effective.

\section{Conclusions}

Administering prophylactic nebulized hypertonic saline to mechanically ventilated children did not improve clinically relevant outcomes, including duration of mechanical ventilation or stay. Wheezing after hypertonic saline treatment was rare, and concurrent bronchodilator therapy may not be needed if hypertonic saline is used. Systemic oxygen desaturation also occurred infrequently but should be anticipated and addressed promptly. Larger randomized control trials are needed to confirm our results.

\section{ACKNOWLEDGMENTS}

We thank Drs James Chmiel, Benjamin Gaston, and Alexandre Rotta for serving on the Data Safety Monitoring Board of this study. We also thank the nurses and respiratory therapists of the Rainbow Babies pediatric ICU for all of their help in the performance of this study.

\section{REFERENCES}

1. Rivera R, Tibballs J. Complications of endotracheal intubation and mechanical ventilation in infants and children. Crit Care Med 1992; 20(2):193-199.

2. Payen V, Jouvet P, Lacroix J, Ducruet T, Gauvin F. Risk factors associated with increased length of mechanical ventilation in children. Pediatr Crit Care Med 2012;13(2):152-157.

3. Branson RD. Secretion management in the mechanically ventilated patient. Respir Care 2007;52(10):1328-1342; discussion 1342-1327. 
4. Kallet RH. Adjunct therapies during mechanical ventilation: airway clearance techniques, therapeutic aerosols, and gases. Respir Care 2013;58(6):1053-1073.

5. National Heart, Lung, and Blood Institute Acute Respiratory Distress Syndrome (ARDS) Clinical Trials Network, Wiedemann HP, Wheeler AP, Bernard GR, Thompson BT, Hayden D. Comparison of two fluid-management strategies in acute lung injury. N Engl $\mathrm{J}$ Med 2006;354(24):2564-2575.

6. Elkins MR, Robinson M, Rose BR, Harbour C, Moriarty CP, Marks $\mathrm{GB}$, et al. A controlled trial of long-term inhaled hypertonic saline in patients with cystic fibrosis. N Engl J Med 2006;354(3):229-240.

7. Zhang L, Mendoza-Sassi RA, Wainwright C, Klassen TP. Nebulised hypertonic saline solution for acute bronchiolitis in infants. Cochrane Database Syst Rev 2013;(7):CD006458.

8. Riethmueller J, Borth-Bruhns T, Kumpf M, Vonthein R, Wiskirchen $\mathrm{J}$, Stern M, et al. Recombinant human deoxyribonuclease shortens ventilation time in young, mechanically ventilated children. Pediatr Pulmonol 2006;41(1):61-66.

9. Dilmen U, Karagol BS, Oguz SS. Nebulized hypertonic saline and recombinant human DNase in the treatment of pulmonary atelectasis in newborns. Pediatr Int 2011;53(3):328-331.

10. Prodhan P, Greenberg B, Bhutta AT, Hyde C, Vankatesan A, Imamura $\mathrm{M}$, et al. Recombinant human deoxyribonuclease improves atelectasis in mechanically ventilated children with cardiac disease. Congenit Heart Dis 2009;4(3):166-173.

11. Rubin BK. Secretion properties, clearance, and therapy in airway disease. Transl Respir Med 2014;2:6.

12. Elkins MR, Bye PT. Mechanisms and applications of hypertonic saline. J R Soc Med 2011;104(Suppl 1):S2-S5.
13. Kishioka C, Okamoto K, Kim JS, Rubin BK. Hyperosmolar solutions stimulate mucus secretion in the ferret trachea. Chest 2003; 124(1):306-313.

14. Gould NS, Gauthier S, Kariya CT, Min E, Huang J, Brian DJ. Hypertonic saline increases lung epithelial lining fluid glutathione and thiocyanate: two protective CFTR-dependent thiols against oxidative injury. Respir Res 2010;11:119.

15. Pollack MM, Patel KM, Ruttimann UE. PRISM III: an updated Pediatric Risk of Mortality score. Crit Care Med 1996;24(5):743-752.

16. Youness HA, Mathews K, Elya MK, Kinasewitz GT, Keddissi JI. Dornase $\alpha$ compared to hypertonic saline for lung atelectasis in critically ill patients. J Aerosol Med Pulm Drug Deliv 2012;25(6): 342-348.

17. Altunhan H, Annagür A, Pekcan S, Ors R, Koç H. Comparing the efficacy of nebulizer recombinant human DNase and hypertonic saline as monotherapy and combined treatment in the treatment of persistent atelectasis in mechanically ventilated newborns. Pediatr Int 2012;54(1):131-136.

18. Snoek AP, Brierley J. Mucolytics for intubated asthmatic children: a national survey of United kingdom paediatric intensive care consultants. Crit Care Res Pract 2015;2015:396107.

19. Schoeffel RE, Anderson SD, Altounyan RE. Bronchial hyperreactivity in response to inhalation of ultrasonically nebulised solutions of distilled water and saline. Br Med J 1981;283(6302): 1285-1287.

20. Ralston S, Hill V, Martinez M. Nebulized hypertonic saline without adjunctive bronchodilators for children with bronchiolitis. Pediatrics 2010;126(3):e520-e525.

This article is approved for Continuing Respiratory Care Education credit. For information and to obtain your CRCE

(free to AARC members) visit www.rcjournal.com

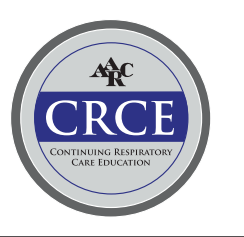

